\title{
Refractive index sensor based on a solid-core photonic crystal fiber interferometer
}

\author{
Nawras Ali Salman and Hanan J. Taher \\ Institute of Laser for Postgraduate Studies, University of Baghdad \\ E-mail: nawras1988@yahoo.com
}

\begin{abstract}
Photonic crystal fiber interferometers are widely used for sensing applications. In this work, solid core-Photonic crystal fiber based on Mach-Zehnder modal interferometer for sensing refractive index was presented. The general structure of sensor applied by splicing short lengths of PCF in both sides with conventional single mode fiber (SMF-28). To apply modal interferometer theory; collapsing technique based on fusion splicing used to excite higher order modes $\left(\mathrm{LP}_{01}\right.$ and $\left.\mathrm{LP}_{11}\right)$. Laser diode $(1550 \mathrm{~nm})$ has been used as a pump light source. Where a high sensitive optical spectrum analyzer (OSA) was used to monitor and record the transmitted. The experimental work shows that the interference spectrum of Photonic crystal fiber interferometer exhibits good sensitivity to refractive index variations. The response of the PCFI is observed for a range of refractive index values from (1.33 to 1.38), the position of the interference peaks is found to be shifted to longer wavelength with refractive index increasing. A different length of PCFs $(2,3,4) \mathrm{cm}$ were used, and the maximum refractive index sensitivity of $(7.5 \mathrm{pm} / \mathrm{RIU})$ is achieved with a PCF length of $4 \mathrm{~cm}$. This refractive index sensor has distinguished properties as that it small size, high sensitivity, fast response time, design flexibility, and immunity to electromagnetic interference.
\end{abstract}

Key words

Refractive Index (RI), interferometer, sensitivity, wavelength shift, Photonic Crystal Fiber (PCF).

Article info.

Received: Feb. 2017 Accepted: Apr. 2017 Published: Sep. 2017

\section{بناء متحسس لمعامل الانكسار المستتد على مقياس التذاخل الليف بصري بلوري ذو القلب الصلب نورس علي سلمان و حنان جعفر طاهر$$
\text { معهد الليزر للارسات العليا، جامعة بغداد }
$$

الخلاصة

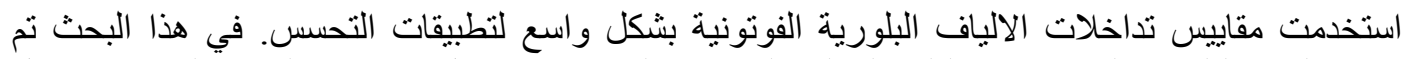

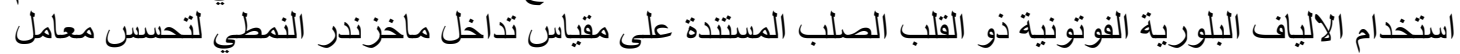

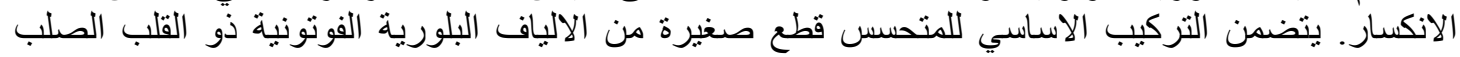

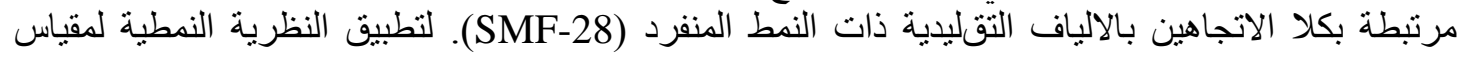

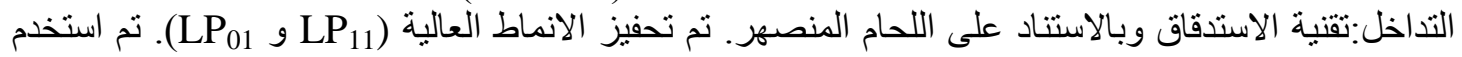

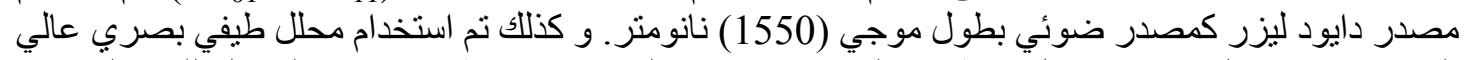

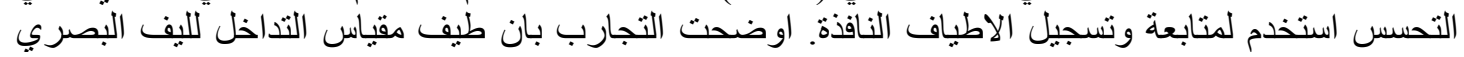

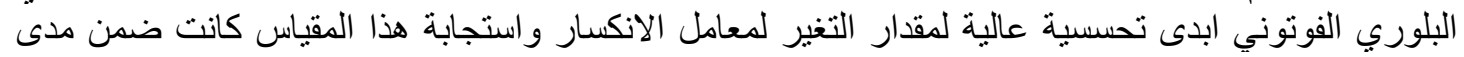

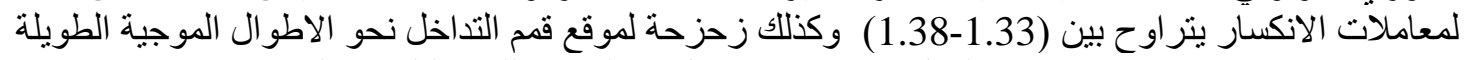

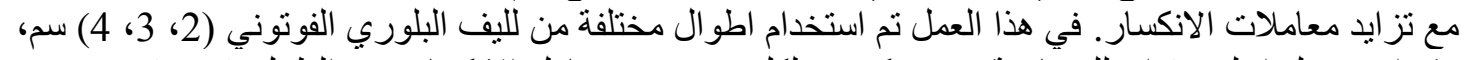

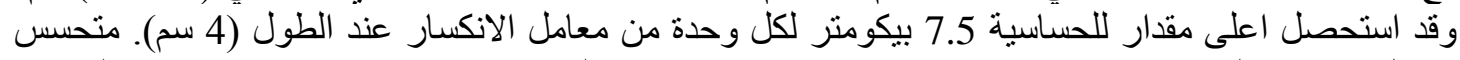

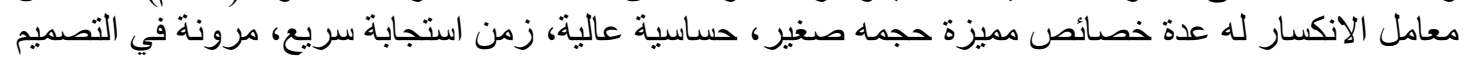

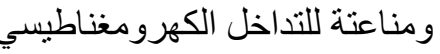




\section{Introduction}

Refractive index is one of the main properties of a material Hence, its accurate measuring is crucial in many applications such as industrial processing and analysis of different biological or chemical specimens [1]. Liquid refractive index measurement based on optical method, has two method optical path measurement method and optical fiber measurement method. Method optical path measurement is a prevalent method but optical path structure is complex and not stable. The structure of optical fiber measurement is simple and is the main method for refractive index measurement. The optical fiber sensor based on Mach-Zehnder interferometer has advantages of fabrication simple and measuring proper. In 2008, Tain proposed single mode fiber MachZehnder interferometer it has advantages of simple fabrication and low cost but the refractive index sensitivity is low [2].

In recent years, Photonic crystal fibers (PCFs), which are also called microstructured optical fibers or holey fiber. The appearance of photonic crystal fibers (PCFs) in 1996 was a breakthrough in fiber optic technology given that these fibers not only had unprecedented properties as they could overcome many limitations intrinsic to standard optical fibers Photonic crystal fiber geometry is characterized by a periodic arrangement of air holes running along the entire length of the fiber, centered on a solid or hollow core. a solid silica core surrounded by air-silica cladding, where the light guiding mechanism is provided by means of a Modified Total Internal Reflection (MTIR), or a hollow core surrounded by air-silica cladding, where the light-guiding mechanism is based on the Photonic Band Gap (PBG) [3, 4]. The interferometers use a pair of collapsed zones separated by a few centimeters is suitable for sensing applications for they offer many advantages such as robustness, low insertion losses and relatively simple fabrication. The operation principle of the PCF interferometers built via micro-holes collapsing is based on the excitation and recombination of two modes [5]. In this paper we proposed and study a kind of Mach- Zehnder interferometer refractive index sensor which based on splicing a short length solid -core photonic crystal fiber between two single -mode fiber. This form a transmission - type PCFI. The research show that the sensitivity sensor structure is with good linearity and repeatability.

\section{Experimental}

The fabrication of the refractive index sensor, PCF (LMA-10) with different lengths of this fiber (2, 3 and 4) $\mathrm{cm}$ used in this experiment spliced with two conventional optical fiber in both sides using fiber splicer FSM- 60S. The PCF (LMA-10) as a sensor head (fabricated in NKT Photonics) consisting of a solid core surrounded by four ring of air holes arranged in a hexagonal pattern around a solid silica core, as shown in Fig.1, the fiber has a core size diameter of $(10 \mu \mathrm{m})$, air-holes with an average diameter of $(3.1 \mu \mathrm{m})$, the average separation between the voids is $(6.6 \mu \mathrm{m})$ and outer diameter of $(125 \mu \mathrm{m})$ dimensions of the PCF (LMA10) alignment and splicing with the SMF with a splicing machine, and due to mode-field diameter mismatch compared to other PCFs, the loss was minimize $[6,7]$. 


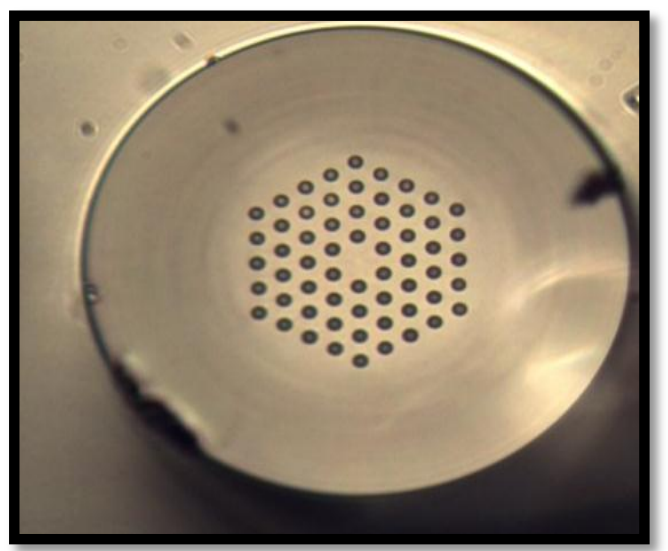

Fig. 1: Cross section of PCF (LMA-10).

During the splicing process and due to surface tension, the air- holes of the PCF collapse with in microscopic length was $\sim 300 \mu \mathrm{m}$, as shown in Fig.2. The fully collapsed air holes of the PCF at the first splicing point allow the coupling of PCF core and cladding modes propagate through same wave guided and then they are subsequently recombined by second splicing point that makes a MachZehnder interferometer [8]. Fig.3 shows the setup of the refractive index sensor based on PCFI, laser source $(1550 \mathrm{~nm})$ is connected to PCFI, and light that transmitted through optical spectrum analyzer (OSA) was used as a recorder of the transmission spectra.

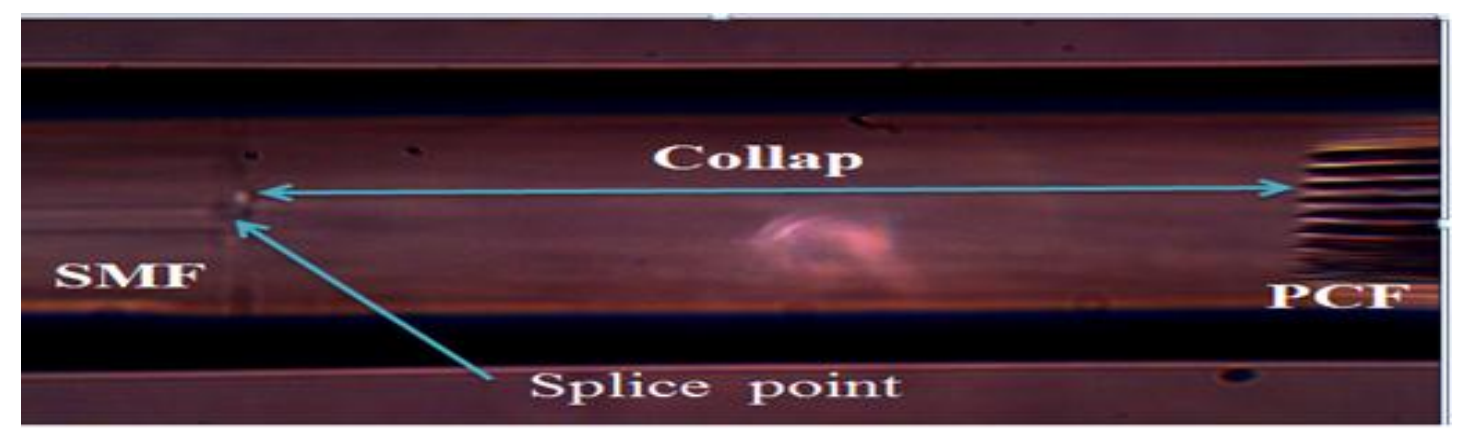

Fig. 2: Microscope image of the splice zone between PCF (LMA-10), on the right, and the $S M F$, on the left the collapsed length $\sim 300 \mu m$.

In our experiments the concentration of $\mathrm{NaCl}$ solution was $(0 \%, 5 \%, 10 \%, 15 \%, 20 \%$ and $25 \%)$ respectively, are used as solution to evaluate the sensitivity of our sensor. Different lengths of Photonic crystal fiber used to show the sensitivity dependence.

The specific experimental operations are as follows:

1- Connecting the light source diode laser $(1550 \mathrm{~nm})$-optical fiber sensing head- optical spectrum analyzer (OSA).
2- The spectrum of fiber sensing head in the (air) is measured and saved as a reference spectrum.

3- The distilled water is injected in to tube with syringe, and then measured and saved the spectrum.

4- Liquid is removed from tube and drying tube with compressed air, until the spectra the same as the reference spectra in the air.

5- The interference spectra of $\mathrm{NaCl}$ solution with different refractive index measurement $(1.34,1.35$, $1.36,1.37$ and 1.38 ) by previous steps. 


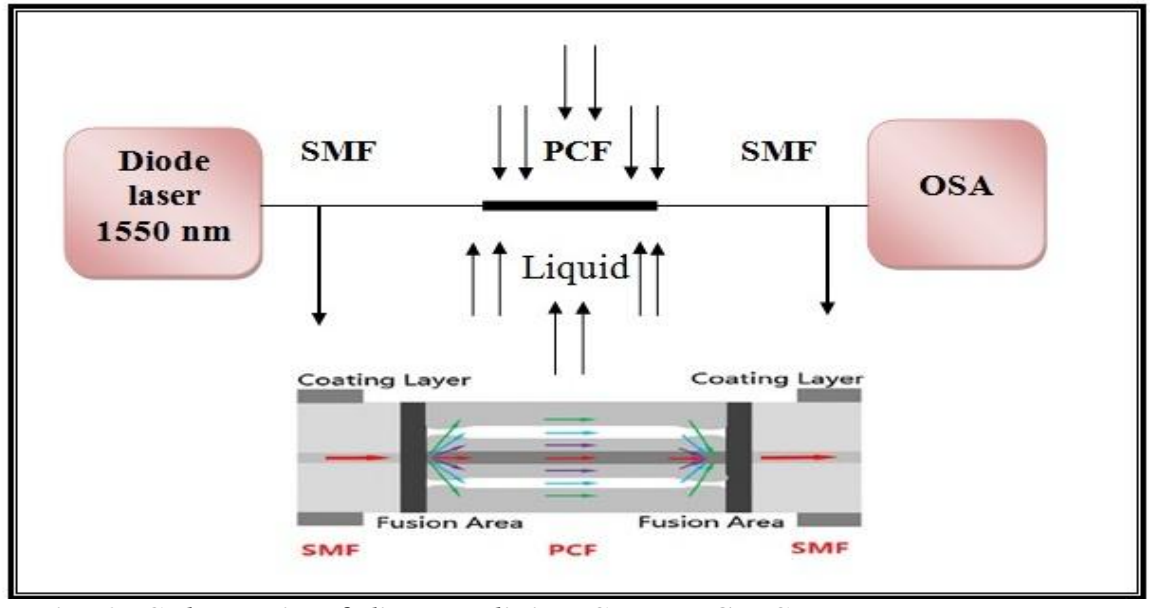

Fig. 3: Schematic of direct splicing SMF-PCF-SMF structure MZI.

\section{Results and discussion}

The ability of PCF interferometer to detect change in the surrounding solution was studied. Different concentrations $(0 \%, 5 \%, 10 \%, 15 \%$, $20 \%$ and $25 \%$ ) of these solution were used to show the minimum detection of the refractive index, the values of the liquids have shown in Table 1.

Table 1: Refractive indices of different concentrations of NaCL solution $(\lambda=1500 \mathrm{~nm})$.

\begin{tabular}{|c|c|c|c|c|c|c|}
\hline Concentration\% & 0 & 5 & 10 & 15 & 20 & 25 \\
\hline Refractive index & 1.33 & 1.34 & 1.35 & 1.36 & 1.37 & 1.38 \\
\hline
\end{tabular}

The fully collapsed air- holes of PCF allow the splitting between core and cladding modes that makes a mach-zehnder interferometer. The core mode is guided by core-cladding interface of the fiber; therefore, its propagation is insensitive to the refractive index of the surrounding.

But the cladding mode is guided by cladding-ambient interface; therefore it is directly exposed to the surrounding, the adsorption of our liquids changes the effective refractive index of the cladding modes in the sensor head, and effective refractive index of photonic crystal fiber core unaffected do with surrounding refractive index, $\Delta \mathrm{n}$ is the variation of effective refractive index of photonic crystal fiber cladding along with surrounding refractive index changing can be expressed as[2].

$\Delta_{\text {neff }}=\mathrm{n}_{\text {eff }}{ }^{\text {core }}-\mathrm{n}_{\text {eff }}$ cladding where $\Delta_{\text {neff }}$ is the difference between effective refractive index of fiber core and cladding $\left(\mathrm{n}_{\mathrm{eff}}^{\text {core }}>\mathrm{n}_{\mathrm{eff}}^{\text {cladding }}\right)$.

The interaction of the cladding mode with external refractive index change $\Delta_{\text {neff }}$ Thus, by depositing a liquid on the PCF surface. Consequently causes a change in the phase difference giving rise to a shift of the interference pattern. The phase difference $\varphi$ can be expressed as [2].

$\varphi=\frac{2 \pi \Delta \text { neff L }}{\lambda}$

where $L$ is optical length of the interferometer, $\lambda$ is light wave length, $\Delta_{\text {neff }}$ is the difference between effective refractive index of fiber core and cladding.

As a result, the position of the interference peaks and change, or equivalently the interference pattern shifts [2].

$\lambda m=\frac{2 \Delta \text { neff } \mathrm{L}}{\lambda}$ 
Figs. 4, 5 and 6 show the transmission spectra of different length of PCF interferometer $(2,3,4) \mathrm{cm}$ for sensing with different refractive index.

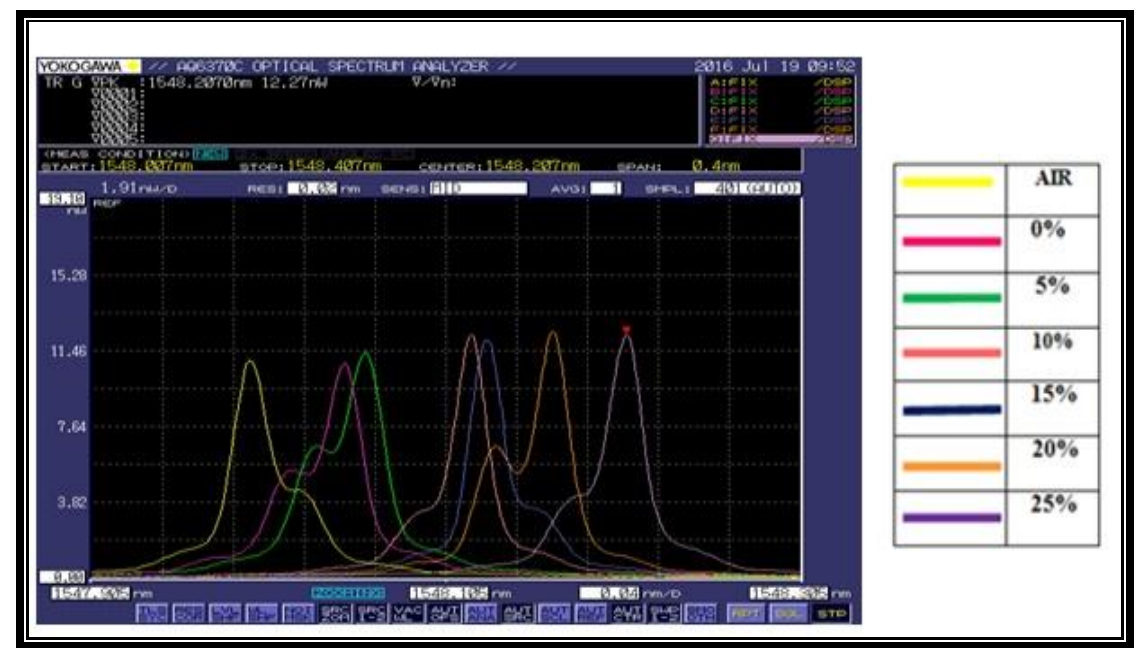

Fig. 4: Transmission spectra of $2 \mathrm{~cm}$ PCF interferometer for sensing with different refractive index.

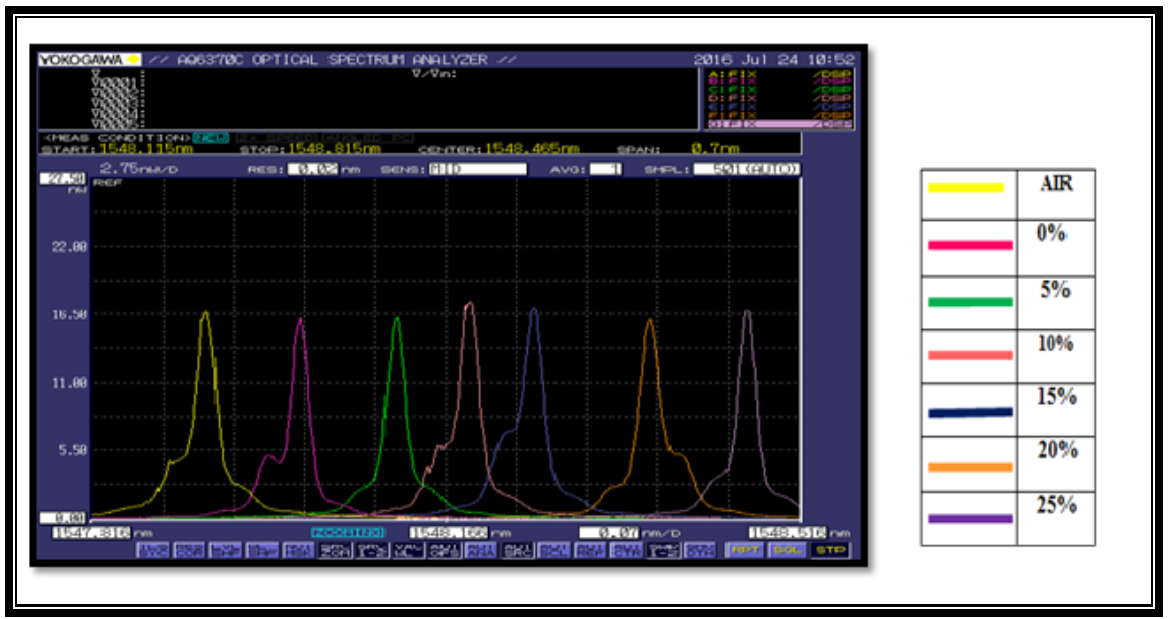

Fig. 5: Transmission spectra of 3cm PCF interferometer for sensing with different rertactive index.

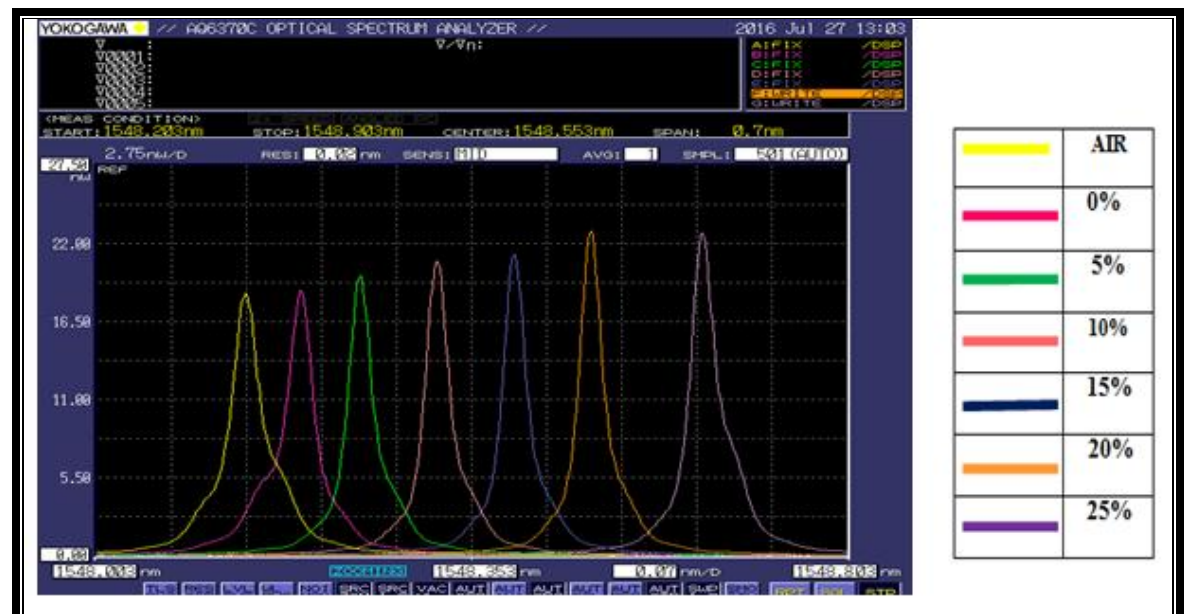

Fig. 6: Transmission spectra of $4 \mathrm{~cm}$ PCF interferometer for sensing with different refractive index. 
The experimental results shown that interference spectrum central wavelength will emerge A red shift as the surrounding refractive index increasing. In refractive index range (1.33-1.38), the sensitivity of refractive index will be increased with increase of photonic crystal fiber length. The sensitivity when PCF length of $4 \mathrm{~cm}$ has sensitivity of $7.4 \mathrm{pm} / \mathrm{RIU}$. The reason of this behaviour is due to for a longer PCF length, the available length for interaction between the adsorbed of solution with the cladding mode is longer which leads to that phase difference obtained between the interfering modes will be longer and leads to the interference pattern shifts therefore sensitivity of refractive index will be increased.

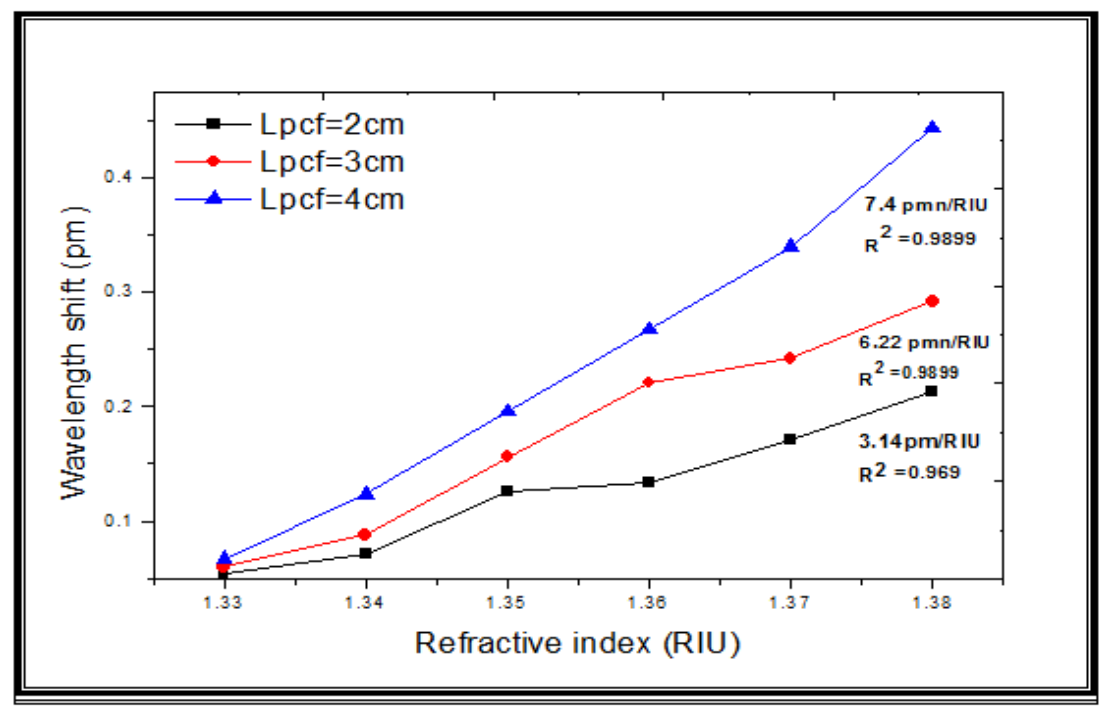

Fig.7: Measuring sensitivity of direct splicing SMF-PCF-SMF Mach-Zehnder interferometer at different PCF Length.

From the previous Fig.7 the sensitivity of refractive index sensor calculated from linear fitting of different refractive index versus wavelength shift at different photonic crystal fiber length and the slope represents refractive index measurement sensitivity. When photonic crystal fiber length is $(2,3$ and 4) $\mathrm{cm}$, the refractive index measurement sensitivity is $(3.14,6.22$ and 7.4) $\mathrm{pm} / \mathrm{RIU}$ respectively and the corresponding fitting degree $\left(\mathrm{R}^{2}\right)$ is $0.969,0.9174$, and 0.9899 .

\section{Conclusions}

The paper proposed and studied a type of Mach-Zehnder mode interferometric refractive index sensor which is based on by direct splicing short pieces of solid core photonic crystal fiber (LMA-10) between two section of standard single-mode fibers (SMF-28). The interferometers were completed by making two collapsing points in PCFs. In this paper, relations between refractive index measurement sensitivity and photonic crystal fiber length and are studied through experiments. The interference spectrum central wavelength will emerge red shift as the surrounding refractive index increasing in refractive index range (1.33-1.38). The sensitivity will be increase with the increase of photonic crystal fiber length the maximum sensitivity of the sensor with photonic crystal fiber length of $4 \mathrm{~cm}$ was $7.4 \mathrm{pm} / \mathrm{RIU}$. The minimum sensitivity of the sensor with photonic crystal fiber length of $2 \mathrm{~cm}$ was $3.4 \mathrm{pm} / \mathrm{RIU}$. 


\section{References}

[1] G. Rajan, S. Mathew, Farrell Dublin Institute of Technology, 13, 42 (2010) 1-10.

[2] Q. Wang, L. Kong, Y. Dang, F.Xia, Y. Zhang, Y. Zhao, H. Hu, J. Li, Sensors and Actuators, 225 (2016) 213-220.

[3] A. MPinto, M. Lopez-Amo, Journal of Sensors, 46, 19 (2012) 1-21.

[4] F. Poli, A. Cucinotta, S. Selleri "Photonic Crystal Fibers Properties a Applications" 1st Edition, 2007.
[5] J.N. Wang, J.L. Tang, Sensors, 12 (2012) 2974-2995.

[6] L. Xiao, MS. Demokan, W. Jin, Y. Wang, CL. Zhao, Wave Technology, 25, 11 (2007) 3563-3574.

[7] B.H. Lee, Y.H. Kim, K.S. Park, JB. Eom, MJ. Kim, B.S. Rho, H.Y. Choi, Sensors, 12 (2012) 2467-2476.

[8] R. Mehra, J .Tripathi, International Journal of Computer Applications, (2014) 31-36. 